\title{
Réduction a priori de modèles thermomécaniques
}

\author{
An a priori model reduction method for thermomechanical problems
}

\section{David Ryckelynck}

Laboratoire de mécanique des systèmes et des procédés, UMR CNRS-ENSAM-ESEM, École nationale supérieure d'Arts et Métiers, 151, boulevard de l'hôpital, 75013 Paris, France

Résumé La méthode de réduction proposée nécessite aucun calcul préalable de l'état de la structure. Le résidu, défini sur tout l'intervalle de temps, des équations obtenues par la méthode des éléments finis et le développement de Karhunen-Loève permettent de définir un faible nombre de fonctions de base pour la représentation spatiale des champs recherchés. Un algorithme non-incrémental, issu de la méthode LATIN, permet de déterminer ces fonctions de base. Le caractère non-incrémental de l'approche garantit la validité du modèle de taille réduite sur un intervalle de temps recouvrant de fortes évolutions de l'état de la structure.

solides et structures / réduction de modèle / sous-espace de Krylov / développement de Karhunen-Loève / approche non-incrémentale / contact

\begin{abstract}
A model reduction method is proposed for finite element models. A previous computation of the state of the structure is not necessary. Residuals defined over the entire time interval and the KarhunenLoève method provide basis functions. A non-incremental algorithm, from the LATIN method, is used to compute this basis functions. Because of the non-incremental feature, the reduced order model is representative for a large evolution of the state of the structure.
\end{abstract}

solids and structures / model reduction / Karhunen-Loève expansion / Krylov subspace / non-incremental approach / contact

\section{Abridged English version}

Numerical simulation of machining processes and metal forming processes need the analysis of timedependant and nonlinear thermomechanical problems. Finite element modelling of such processes uses generally a large number of variables to describe the state of the structure. The aim of the model reduction is to define a transformation matrix to get few basis functions to describe the spatially distributed state, with few degree of freedom. For nonlinear time-dependant problems we can distinguish an a posteriori approach based on Karhunen-Loève expansion [1,2] and an a priori approach based on the Krylov subspace [3-5]. With the Karhunen-Loève method, the basis functions are generated by a state snapshots taken at various 
time instants. Principal directions of the space defined by the snapshots are taken as the basis functions. In the framework of nonlinear problems, the Krylov subspace is mainly used in order to reduce the cost of the incremental computation of the state by using few unknowns. At a given time step, the basis functions are built with the residual of the nonlinear equations of the finite element problem and a linear system defined to correct the approximate state forecasted at the end of the time step. The basis functions can be used to simplify the computation of the state for several time increments [3]. In this paper we propose to build the basis functions of the reduced-order model with residuals checking the nonlinear equations on the entire time interval. A thermomechanical problem, involving small strain small displacement, elasticity, and contact producing thermo-mechanical coupling, is considered. The structure $\Sigma$ is divided into subdomain. For each sub-domain $\Omega$, the global equations are linear (1), (2). The local equations concern the description of the constitutive law, the boundary condition, .... The improvement of the reduced-order model is made simultaneously with the correction of the approximate state used to determine the residuals. To obtain a decrease of the residuals, those corrections must be defined over the entire time interval. Hence, the proposed approach is necessarily a non-incremental one. The residuals and the corrections of the state are defined by the LATIN method [6,7]. The algorithm is defined by a choice of search directions (5). The correction stage is defined by two linear problems (3) and (4). The approximate boundary conditions $\underline{U}_{n+1 / 2}, \underline{F}_{n+1 / 2}, \phi_{n+1 / 2}$ et $T_{n+1 / 2}$ defined over $\partial \Omega$ are obtained from the local equations of the boundary conditions and the search directions. Residuals are defined for the displacement field $\underline{W}_{U}$, the stresses $\left(\mathbf{W}_{\sigma}\right.$, $\underline{W}_{F}$ ), for the temperature field $W_{T}$ and $W_{\phi}$ for the heat transfer over $\partial \Omega$. As proposed by Ladevèze in [7], they are obtained by a weak form of the equations of the correction stage (3), (4) and a convenient choice of scalars time functions $\lambda^{\text {me }}$ and $\lambda^{\text {th }}$. Several correction stages of the approximate state must be considered in order to obtain residuals which span a sufficient large subspace to define the reduced-order model. The subspace of the residuals is called the LATIN subspace. Obviously, the basis functions contained in matrix the $\mathbf{A}_{x}(x=U, \sigma, T, \phi)$ are used to compute the corrections of the state (6). Bussy [8] has already proposed the same kind of subspace. But it was used only for the computation of a part of the correction of the variables. We propose to use this subspace to construct a reduced-order model. During the correction stages the basis functions we look for are extracted from the LATIN subspace thanks to the Karhunen-Loève expansion, by selecting the most significant patterns of the known approximate state (8), (7).

A 2D example illustrates the capability of the model reduction technique. It corresponds to a simple casting problem (Fig. 1). The material of the part is a kind of aluminum and the mould material is a kind of steel. We obtain a reduced-order model defined for large nonlinear transformations during all the cooling of the part. Nonlinearities are due to the thermal contraction of the part and thermal expansion of the mould which modify the thermomechanical contact conditions between the part and the mould. An accurate prediction is obtained with few basis functions obtained with 80 correction stages (Table 1). The proposed reduction method should be interesting to extract a reduced-order model from a very fine FE description of complex nonlinear problems.

\section{Introduction}

La simulation de procédés de fabrication, comme la coupe des métaux ou la fonderie, nécessite l'étude de problèmes de thermomécanique non-linéaires dépendants du temps. La modélisation de tels procédés conduit généralement à des modèles issus de la méthode des éléments finis où l'état de la structure est représenté par un très grand nombre de variables. L'enjeu de la réduction de ces modèles est de définir un changement de base (un sous-espace), pour la représentation spatiale des variables d'état, qui permette d'utiliser un faible nombre de degrés de liberté.

Pour les problèmes non-linéaires dépendants du temps, deux d'approches peuvent être utilisées. L'une, a posteriori, est bâtie sur la connaissance de la prévision fournie par la méthode des éléments finis et 
l'autre, a priori, n'exploite pas cette prévision. La première consiste à construire un sous-espace associé au développement de Karhunen-Loève [1,2] et l'autre exploite le sous-espace de Krylov [3-5]. Le développement de Karhunen-Loève permet d'extraire de la prévision connue des directions principales qui caractérisent les phénomènes les plus significatifs de l'évolution de l'état de la structure. Le sousespace de Krylov est surtout utilisé, dans le cadre des problèmes non-linéaires, pour réduire le coût du calcul incrémental d'une solution approchée en exploitant un nombre réduit d'inconnues. À un instant $t_{i}$, les fonctions de base sont construites en exploitant le résidu des équations du problème éléments finis nonlinéaire et un système linéaire défini pour corriger les approximations de l'état du système à l'instant $t_{i}$. Les fonctions de base ainsi définies peuvent être utilisées pour simplifier le calcul d'une solution approchée sur plusieurs pas de temps [3].

Dans cet article, nous proposons de construire les fonctions de base du modèle de taille réduite en exploitant des résidus caractérisant la vérification sur tout l'intervalle de temps des équations du problème non-linéaire. L'amélioration du modèle de taille réduite est obtenue simultanément avec la correction de la solution approchée utilisée pour évaluer les résidus. Pour obtenir une décroissance des résidus considérés, les corrections apportées à la solution approchée sont nécessairement définies sur tout l'intervalle de temps. L'approche est donc non-incrémentale. Les résidus et les corrections de l'état du système sont déterminés à l'aide de la méthode LATIN [6,7]. Plusieurs étapes de correction de la solution approchée doivent être envisagées pour obtenir une suite de résidus qui engendrent un sous-espace suffisamment représentatif pour définir le modèle de taille réduite. Le sous-espace ainsi engendré est appelé sous-espace LATIN. Au cours des étapes de correction, des fonctions de base sont extraites du sous-espace LATIN à l'aide de la méthode de Karhunen-Loève. Nous obtenons ainsi un sous-espace appelé sous-espace LATIN-KL.

Cette méthode de réduction de modèle est consécutive aux travaux de Ladevèze [7] et de Bussy [8] sur la représentation des corrections construites par la méthode LATIN. P. Bussy est le premier a avoir proposé d'utiliser un sous-espace connu, pour effectuer une correction préliminaire de la prévision en cours de construction. Ce sous-espace correspond au sous-espace LATIN. Nous avons constaté que cette correction préliminaire est suffisante pour permettre à elle seule la convergence d'un algorithme LATIN, sous réserve que le sous-espace LATIN soit bien adapté au problème traité. Ceci a été illustré pour des problèmes de viscoplasticité [9]. La performance de cette approche permet de proposer dans cet article une véritable méthode de réduction de modèle, ce qui est bien plus qu'une technique de calcul de correction pour la méthode LATIN.

\section{Définition de résidus et formulation de l'étape de correction}

Pour simplifier notre approche, nous supposons que les déplacements et les déformations sont petits. De plus, dans un souci de concision, nous ne présentons dans cet article que le cas des matériaux élastiques, sans remettre en cause les idées principales de l'approche proposée. La structure $\Sigma$ est découpée en sousdomaines $\Omega$ qui ne font pas intervenir de phénomènes de contact en leur sein. La méthode LATIN est bâtie sur les trois principes suivants [6] :

- P1 : séparation des difficultés, partition des équations en deux groupes : un groupe d'équations locales en variable d'espace, éventuellement non-linéaires, et un groupe d'équations linéaires, éventuellement globales en variable d'espace;

- P2 : approche itérative à deux étapes où, à chaque itération, on construit une solution de chaque groupe d'équations;

- P3 : utilisation d'approximations temps-espace ad hoc, à contenu «mécanique », pour le traitement du problème global défini sur $\Sigma \times\left[0, t_{f}\right]$.

Une analyse de la nature des équations du problème de référence est donc nécessaire afin de définir leur caractère local ou global, linéaire ou non-linéaire, et dans le cas qui nous intéresse leur caractère couplé ou découplé. Or, en éliminant certaines variables intermédiaires, la nature des équations peut être modifiée. En conservant comme inconnues la dissipation mécanique $r$ définie dans le volume et la contrainte $\sigma$, le 
taux de variation de l'énergie interne $\dot{e}$ et le flux de chaleur $q$, les équations globales issues des principes de conservation sont linéaires et découplées. De plus, en introduisant comme inconnues les efforts extérieurs $\underline{F}$ et les flux de chaleur $\phi$ s'exerçant sur la frontière de chaque sous-domaine $\Omega$, les équations de conservations définie sur chaque sous-domaines sont elles aussi découplées. Ce sont ces équations qui forment le groupe des équations linéaires :

$$
\begin{aligned}
& \left.\left.\int_{\Omega} \operatorname{Tr}\left[\boldsymbol{\varepsilon}\left(\underline{U}^{*}\right) \boldsymbol{\sigma}\right] \mathrm{d} \Omega=\int_{\partial \Omega} \underline{U^{*}} \cdot \underline{F} \mathrm{~d} S \quad \forall \underline{U}^{*} \text { régulier, } \forall \Omega \in \Sigma, \forall t \in\right] 0, t_{f}\right] \\
& \left.\left.\int_{\Omega} T^{*} \dot{e} \mathrm{~d} \Omega-\int_{\Omega} \underline{\operatorname{grad}}\left(T^{*}\right) \underline{q} \mathrm{~d} \Omega=-\int_{\partial \Omega} T^{*} \phi \mathrm{d} S+\int_{\Omega} T^{*} r \mathrm{~d} \Omega \quad \forall T^{*} \text { regulier, } \forall \Omega \in \Sigma, \forall t \in\right] 0, t_{f}\right]
\end{aligned}
$$

Les équations complémentaires du problème de référence sont locales. Elles définissent le comportement thermo-élastique de la matière dans le volume, l'état initial de la matière, le comportement d'interface entre les sous-domaines et les conditions aux limites sur $\partial \Sigma$. Elles forment le deuxième groupe d'équations. Une solution approchée unique est obtenue à chaque étape de l'algorithme en exploitant des directions de recherche. Pour les équations d'interface nous avons utilisé des directions de recherche similaires à celles proposées dans le cadre de problèmes de contact [10]. Ces directions sont définies par les paramètres $\widehat{k}^{\text {me }}$ et $\widehat{h}^{\text {th }}$. En ce qui concerne les phénomènes mis en jeu dans le volume de chaque sous-domaine l'algorithme ressemble à une méthode de point fixe. Pour améliorer la prévision de l'état de la structure, nous choisissons comme inconnues principales le champ de déplacements $\underline{U}$ et le champ de températures $T$. En supposant connue une prévision de l'état de la structure $\left(\underline{U}_{n}, T_{n}, \ldots\right)$ définie sur $\left[0, t_{f}\right]$, la formulation de la $(n+1)$ ième étape de correction est composée du problème de mécanique et du problème de thermique suivants :

$\square$ problème mécanique pour chaque sous-domaine $\Omega \in \Sigma$, trouver $\underline{U}$ régulier tel que :

$$
\begin{aligned}
\int_{\Omega} \operatorname{Tr}\left[\boldsymbol{\varepsilon}\left(\underline{U}^{*}\right) \mathbf{K}\left(T_{n}\right)\left(\boldsymbol{\varepsilon}(\underline{U})-\alpha\left(T_{n}-T_{\mathrm{ini}}\right)\right)\right] \mathrm{d} \Omega \\
\left.\left.\quad=\int_{\partial \Omega} \underline{U}^{*} \cdot\left(\underline{F}_{n+1 / 2}-\widehat{k}^{\mathrm{me}}\left(\underline{U}-\underline{U}_{n+1 / 2}\right)\right) \mathrm{d} S \quad \forall \underline{U}^{*} \text { régulier, } \forall t \in\right] 0, t_{f}\right]
\end{aligned}
$$

où $T_{\text {ini }}$ est le champ de température à l'instant initial ;

$\square$ problème de thermique, trouver $T$ régulier tel que :

$$
\begin{aligned}
& \int_{\Omega} T^{*} \rho c\left(T_{n}\right) \dot{T} \mathrm{~d} \Omega+\int_{\Omega} \underline{\operatorname{grad}}\left(T^{*}\right) k\left(T_{n}\right) \underline{\operatorname{grad}}(T) \mathrm{d} \Omega \\
& \left.\left.\quad=-\int_{\partial \Omega} T^{*}\left(\phi_{n+1 / 2}+\widehat{h}^{\text {th }}\left(T-T_{n+1 / 2}\right)\right) \mathrm{d} S \quad \forall T^{*} \text { regulier, } \forall t \in\right] 0, t_{f}\right]
\end{aligned}
$$

où $\underline{U}_{n+1 / 2}, \underline{F}_{n+1 / 2}, \phi_{n+1 / 2}$ et $T_{n+1 / 2}$ vérifient les équations complémentaires d'interface ou de conditions aux limites, définies sur le bord des sous-domaines, ainsi que les équations suivantes associées à la direction de recherche de l'étape locale :

$$
\left.\left.\underline{F}_{n+1 / 2}=\underline{F}_{n}+\widehat{k}^{\mathrm{me}}\left(\underline{U}_{n+1 / 2}-\underline{U}_{n}\right) \phi_{n+1 / 2}=\phi_{n}-\widehat{h}^{\mathrm{th}}\left(T_{n+1 / 2}-T_{n}\right) \quad \text { sur } \partial \Omega, \forall t \in\right] 0, t_{f}\right]
$$

Pour extraire des résidus du problème de mécanique et du problème de thermique, nous utilisons deux fonctions du temps données $\lambda^{\mathrm{me}}(t)$ et $\lambda^{\text {th }}(t)$ comme cela est proposé dans [7]. Nous obtenons les champs $\underline{W}_{U}, \mathbf{W}_{\sigma}, \underline{W}_{F}, W_{T}$ et $W_{\phi}$ tel que :

$$
\begin{aligned}
\underline{U} & =\underline{U}_{n}+\lambda^{\mathrm{me}}(t) \underline{W}_{U}, \quad \sigma=\sigma_{n}+\lambda^{\mathrm{me}}(t) \mathbf{W}_{\sigma}, \quad \underline{F}=\underline{F}_{n}+\lambda^{\mathrm{me}}(t) \underline{W}_{F} \\
T & =T_{n}+\lambda^{\mathrm{th}}(t) W_{T}, \quad \phi=\phi_{n}+\lambda^{\mathrm{th}}(t) W_{\phi}
\end{aligned}
$$

Les fonctions du temps $\lambda^{\text {me }}$ et $\lambda^{\text {th }}$ sont choisies pour avoir une intensité décroissante et un signe permettant une accumulation des résidus sur $\left[0, t_{f}\right]$, comme cela a été indiqué dans [9]. Les champs $\underline{W}_{U}, \mathbf{W}_{\sigma}, W_{T}$ et $W_{\phi}$ déterminés par les étapes de correction successives définissent le sous-espace LATIN. 


\section{Construction du sous-espace LATIN-KL}

Le modèle de taille réduite mis en place consiste à représenter les inconnues $\underline{x}_{n+1}(x=U, \sigma, T, \phi) \mathrm{du}$ problème de thermomécanique à l'aide des matrices de fonctions de base $\mathbf{A}_{x}$ du sous-espace LATIN-KL, tel que :

$$
\underline{x}_{n+1}=\mathbf{A}_{x} \underline{a}_{x n+1}(t)+\underline{x}_{0} \quad \text { avec } \underline{a}_{x n+1}(0)=0
$$

où $\underline{x}_{0}$ est la valeur à l'instant initial de la variable considérée. Les valeurs corrigées des degrés de liberté du modèle de taille réduite $\underline{a}_{x+1}(x=U, \sigma, T, \phi)$ sont données par la formulation variationnelle des équations de l'étape de correction. Un découpage en pas de temps de l'intervalle $\left[0, t_{f}\right]$ et le schéma d'Euler implicite sont utilisés pour la résolution des équations différentielles en temps. Les champs $\underline{W}_{x}(x=U, \sigma, T, \phi)$ peuvent être utilisés pour définir les matrices $\mathbf{A}_{x}$. Mais ils n'ont pas de sens physique fort et leur nombre dépend du nombre d'étape de correction effectuées. Pour améliorer ces vecteurs de base, nous utilisons le développement de Karhunen-Loève [1].

Soit $\mathbf{X}$ la matrice des fonctions de base du sous-espace LATIN tel que $: \underline{x}(t)=\mathbf{X} \underline{\widetilde{x}}(t)+\underline{x}_{0}$. La suite des taux de variation $\underline{\tilde{\tilde{x}}}$, pas de temps par pas de temps, engendre un sous-espace de l'espace LATIN. Nous appliquons le développement de Karhunen-Loève à cette suite de valeurs pour définir les matrices de fonctions de base $\mathbf{A}_{x}$ à l'aide d'une matrice de changement de base $\mathbf{V}$ :

$$
\mathbf{A}_{x}=\mathbf{X} \cdot \mathbf{V}
$$

Les colonnes de $\mathbf{V}$ sont des directions principales $v_{i}$ de l'espace engendré par les valeurs de $\underline{\tilde{\widetilde{x}}}$ sur $\left[0, t_{f}\right]$. Ces directions principales sont définies par le problème aux valeurs propres suivant :

$$
\mathbf{M} \cdot v_{i}=\mu_{i} v_{i} \quad \text { avec } \quad \mathbf{M}=\int_{0}^{t_{f}} \dot{\tilde{\tilde{x}}}(t) \dot{\tilde{\tilde{x}}}^{\mathrm{T}}(t) \mathrm{d} t
$$

Soit $\mu_{\max }$, la plus grande valeur propre de $\mathbf{M}$. Pour ne représenter que les principaux phénomènes, les directions principales que nous sélectionnons pour $\mathbf{V}$ sont les vecteurs propres $v_{i}$ dont la valeur propre associée est supérieure à $10^{-6} \mu_{\max }$.

Afin de conserver la meilleure représentation possible de l'évolution de $x$ sur l'intervalle $\left[0, t_{f}\right]$, nous déterminons une combinaison linéaire $\underline{a}_{x}$ des vecteurs de base de $\mathbf{A}_{x}$ en exploitant l'inverse de MoorePenrose de $\mathbf{V}$. Cette amélioration des vecteurs de base est réalisée après l'étape de correction, pour une étape sur trois. En pratique, nous privilégions les premiers instants de l'intervalle de temps dans l'équation (8) à l'aide d'une pondération.

\section{Exemple numérique}

Pour illustrer la capacité de réduction de modèle qu'offre l'approche proposée, voici l'étude du refroidissement d'une pièce en aluminium (sous-domaine $\Omega$ Fig. $1, T_{\text {ini }}=600^{\circ} \mathrm{C}$ ) moulée dans un moule en acier (sous-domaine $\Omega^{\prime}, T_{\mathrm{ini}}=20^{\circ} \mathrm{C}$ ). Les déplacements sont supposés plans. Les deux sous-domaines sont en contact à l'instant initial. Le frottement est décrit par une loi de Coulomb. Pour modéliser simplement le lien entre le jeu et les conditions de transfert de chaleur, le coefficient de conduction est pondéré par une fonction exponentielle du jeu. La prévision des températures obtenue à la fin du calcul itératif est donnée pour 2 instants particuliers sur la Fig. 1. Les non-linéarités sont issues des conditions de contact et du restreint de la pièce qui provoquent l'apparition de lames d'air modifiant les conditions de transfert thermique. Les maillages utilisés ainsi que les conditions aux limites sont donnés sur la figure ci-dessous (Fig. 1). L'intervalle de temps couvre l'ensemble du refroidissement. Il est découpé en 40 pas de temps.

Peu d'étapes globales de correction (environ 40) permettent d'obtenir les principaux phénomènes mis en jeux au cours du refroidissement. Le nombre de fonctions de base proposées à chaque étape globale est 



Figure 1. Modèle éléments finis et solution approchée obtenue avec 80 étapes de correction.

Figure 1. Mesh and approximate solution obtained with 80 correction stages.

Figure 2. Nombre de fonctions de base pour représenter $T$ dans $\Omega$ au cours de l'amélioration du modèle de taille réduite.

Figure 2. Number of basis fonction for $T$ in $\Omega$ during the improvement of the reduced-order model.

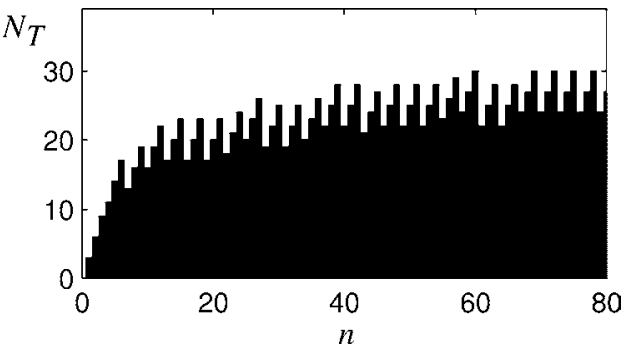

Tableau 1. Nombre d'inconnues par sous-domaine : modèle réduit $(n=80) /$ modèle E.F.

Table 1. Number of variables for each subdomain: reduce order model $(n=80) / E . F$. model.

\begin{tabular}{lcccc}
\hline & $\underline{U}$ & $T$ & $(\sigma, \underline{F})$ & $\phi$ \\
\hline$\Omega$ & $18 / 636$ & $27 / 318$ & $24 / 1647$ & $13 / 47$ \\
$\Omega^{\prime}$ & $34 / 926$ & $27 / 463$ & $42 / 2532$ & $12 / 47$ \\
\hline
\end{tabular}

reporté sur la Fig. 2 pour la représentation des champs de température dans $\Omega$. En pratique, 4 fonctions de bases sont proposées à chaque étape globale pour augmenter la base associée à chaque variable d'état. Pour cela, différentes fonctions $\lambda_{u}$ et $\lambda_{T}$ sont utilisées. La chute du nombre de fonction de base est due à l'étape d'amélioration en exploitant la méthode de Karhunen-Loève. On constate que peu de degrés de liberté sont utilisés par le modèle de taille réduit au cours de sa construction.

Après 80 étapes globales de correction, le modèle de taille réduite est peu modifié. Le nombre d'inconnues du modèle de taille réduite est présenté par grandeur physique et par sous-domaine dans le Tableau 1.

\section{Conclusion}

Un modèle de taille réduite est construit par un algorithme itératif, à partir des équations fournies la méthode des éléments finis. Cette approche algorithmique ne nécessite pas une analyse préalable pour distinguer les principaux phénomènes à décrire. Pour mettre en œuvre l'algorithme itératif, nous avons exploité la méthode LATIN qui laisse une grande liberté quant à la représentation temps-espace des 
variables d'état d'une structure. Les résidus des équations de l'étape globale de l'algorithme LATIN donnent des indications sur la complexité de la solution recherchée. Ils permettent de définir, par itérations successives, un sous-espace LATIN pour la représentation spatiale des inconnues du problème non-linéaire. Les fonctions de base du modèle de taille réduite sont extraites du sous-espace LATIN de façon à bien représenter les phénomènes les plus significatifs, en exploitant le développement de Karhunen-Loève. Grâce à l'approche non-incrémentale, les mêmes fonctions de base sont exploitées pour décrire de fortes évolutions non-linéaires.

\section{Références bibliographiques}

[1] L. Sirovich, Empirical eigenfunctions and low dimensional systems, New Perspectives in Turbuence 5 (1991) 139.

[2] H.-M. Park, W.-S. Jung, The Karhunen-Loève Galerkin method for the inverse natural convection problems, Int. J. Heat Mass Transfer 44 (2001) 155-167.

[3] D.-A. Knoll, P.-R. McHugh, Newton-Krylov methods applied to a system of convection-diffusion-reaction equations, Comput. Phys. Comm. 88 (1995) 141-160.

[4] R. Weiss, A theorical overview of Krylov subspace methods, Appl. Numer. Math. 19 (1995) 207-233.

[5] S. Sundar, B.-K. Bhagavan, S. Prasad, Newton-preconditioned Krylov subspace solvers for system of nonlinear equations: A numerical experiment, Appl. Math. Lett. 14 (2001) 195-200.

[6] P. Ladevèze, Sur une famille d'algorithmes en mécanique des structures, C. R. Acad. Sci. Paris, Série II 300 (2) (1985) 41-44.

[7] P. Ladevèze, Mécanique non linéaire des stuctures, études en mécanique des matériaux et des structures, Hermès, 1996, p. 265.

[8] P. Bussy, P. Rougée, P. Vauchez, The large time increment method for numerical simulation of metal forming processes, in: Proc. NUMETA, Elsevier, 1990, pp. 102-109.

[9] J.-P Pelle, D. Ryckelynck, An efficient adaptive strategy to master the global quality of viscoplastic analysis, Comput. \& Structures 78 (1-3) (2000) 169-184.

[10] L. Champaney, J.-Y. Cognard, D. Dureisseix, P. Ladevèze, Numerical experimentations of parallel strategies in structural non-linear analysis, Calc. Parallèles 8 (2) (1996) 245-249. 